\title{
Toward twist-2 $T$-odd transverse-momentum-dependent gluon distributions: the $f$-type linearity function
}

\author{
Alessandro Bacchetta, ${ }^{a, b}$ Francesco Giovanni Celiberto $^{c, d, e, *}$ and Marco Radici ${ }^{b}$ \\ ${ }^{a}$ Dipartimento di Fisica, Università di Pavia, via Bassi 6, I-27100 Pavia \\ ${ }^{b}$ INFN Sezione di Pavia, via Bassi 6, I-27100 Pavia, Italy \\ ${ }^{c}$ European Centre for Theoretical Studies in Nuclear Physics and Related Areas (ECT*), I-38123 \\ Villazzano, Trento, Italy \\ ${ }^{d}$ Fondazione Bruno Kessler (FBK), I-38123 Povo, Trento, Italy \\ e INFN-TIFPA Trento Institute of Fundamental Physics and Applications, I-38123 Povo, Trento, Italy \\ E-mail: alessandro.bacchetta@unipv.it, fceliberto@ectstar.eu, \\ marco.radici@pv.infn.it
}

We present preliminary results on the leading-twist transverse-momentum-dependent (TMD) gluon linearity function, which is directly connected to nucleon transverse-spin asymmetries originating from the density of linearly-polarized gluons. The function is calculated in a spectatormodel framework for the parent proton and for the $f$-type gauge-link structure. Our work represents a further step toward the definition of a complete set of gluon TMD distributions at twist-2 that can be used to access the gluon dynamics inside nucleons and nuclei at new-generation colliders.

\footnotetext{
*** Particles and Nuclei International Conference - PANIC2021 ***

*** 5 - 10 September, $2021 * * *$

$* * *$ Online $* * *$
}

\footnotetext{
${ }^{*}$ Speaker
} 


\section{Introductory remarks}

The study of the inner structure of nucleons in terms of the distribution of their constituents has always represented a frontier research field in particle physics. The well-established collinear factorization has played an important role in the description of high-energy hadronic and leptohadronic collisions in terms of one-dimensional parton distribution functions (PDFs). There are fundamental questions, however, that are still open and whose answers cannot be made from a purely collinear perspective. To shed light on the origin of proton spin and mass as well as on azimuthal asymmetries determined by the interplay between nucleon and parton polarizations, a three-dimensional vision is required. The transverse-momentum-dependent (TMD) factorization represents the most powerful tool to afford such a tomographic description. At variance with the quark-TMD sector, where significant results have been collected both on the formal and phenomenological sides, gluon TMDs are still a largely unexplored territory. The first classification of (un)polarized TMD gluon densities was done in [1] and then extended in [2-4]. First phenomenological studies were conducted in [5-12]. A remarkable feature that distinguishes TMD densities from their collinear counterparts is the sensitivity to the gauge link, which leads to the well-known TMD process dependence [13-15]. While quark TMDs depend on processes via the [+] and [-] staple links respectively depicting the direction of future- and past-pointing Wilson lines, gluon TMDs depend on combinations of those gauge links, thus bringing to a more diversified modified universality. Two major gluon gauge links appear: the $f$-type (or Weiszäcker-Williams) and the $d$-type (or dipole) ones. The $f$-type $T$-odd gluon-TMD correlator is characterized by the $f_{a b c} \mathrm{QCD}$ color structure, whereas the symmetric $d_{a b c}$ structure emerges in the $d$-type $T$-odd one. It turns out that $f$-type TMDs depend on $[ \pm, \pm]$ gauge links, while $d$-type TMDs depend on $[ \pm, \mp]$ ones. Box-loop gauge links are probed via reactions where multiple color exchanges connect both initial and final states [16], thus generating factorization-breaking effects [17]. The connection between TMD and high-energy factorization was investigated in recent studies on the BFKL unintegrated gluon density [18-34]. Detailed studies on spectator-model quark TMDs in the proton were done in [35, 36]. A common framework was recently built [37] (see also [38, 39]) for all the $T$-even gluon TMDs at twist-2. These functions were calculated in an enhanced spectator model for the parent proton and they encode effective high-energy resummation effects. In [40] we presented a first extension of our framework to $T$-odd gluon TMDs by means of the $f$-type Sivers function, which carries information on the distribution of unpolarized gluons inside transversely polarized protons. In this work we present a preliminary analysis of another $T$-odd gluon TMD, the $f$-type linearity function, which can explain observed single-spin asymmetries originating from the density of linearly-polarized gluons inside transversely polarized protons.

\section{The $f$-type linearity function in a spectator model}

The spectator-model framework relies on the assumption that an incoming nucleon, having mass $M$ and four-momentum $P$, emits a parton with four-momentum $p$, transverse momentum $\boldsymbol{p}_{T}$ and longitudinal fraction $x$, and what remains is considered as an effective on-shell spectator particle having mass $M_{X}$ and spin-1/2. We model the nucleon-gluon-spectator vertex in the following way

$$
Y^{\mu}=\left(g_{1}\left(p^{2}\right) \gamma^{\mu}+g_{2}\left(p^{2}\right) \frac{i}{2 M} \sigma^{\mu v} p_{\nu}\right) \text {. }
$$



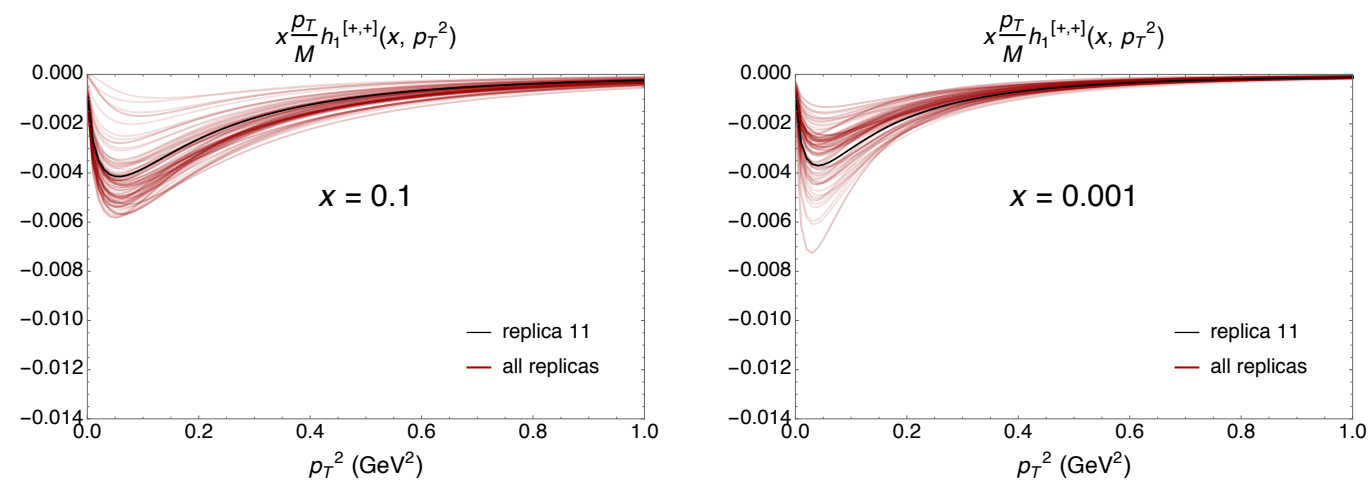

Figure 1: Transverse-momentum dependence of the $[+,+]$ linearity function for $x=10^{-1}$ (left) and $x=10^{-3}$ (right), and at the initial scale $Q_{0}=1.64 \mathrm{GeV}$. Black curve stands for the most representative replica \#11.

In Eq. (1) $g_{1}$ and $g_{2}$ are dipolar functions of $\boldsymbol{p}_{T}^{2}$. Employing dipolar form factors is quite useful, since it permits us to quench gluon-propagator divergences, suppress large- $\boldsymbol{p}_{T}$ effects that are beyond the reach of a genuine TMD description, and cancel logarithmic singularities arising from $\boldsymbol{p}_{T}$-integrated densities. All the leading-twist $T$-even gluon TMDs in the proton were calculated in [37] by defining an enhanced version of the tree-level spectator model. In particular, the $M_{X}$ mass of the colored spin- $1 / 2$ spectator was integrated over a continuous range weighed by a flexible spectral function suited to catch both small- and moderate- $x$ effects (see Eqs. (16) and (17) of [37]). Parameters embodied by the spectral mass and the pure spectator-model correlator were fixed by making a simultaneous fit of our unpolarized and helicity TMD distributions, $f_{1}^{g}$ and $g_{1}^{g}$, to the corresponding collinear PDF functions from NNPDF [41, 42] at the initial scale $Q_{0}=1.64$ $\mathrm{GeV}$. Statistical uncertainties are described via the bootstrap method. The spectator-model $T$ even functions are process-independent, since the tree-level approximation for the gluon correlator does not account for the gauge link. The interference term between two scattering amplitudes with different imaginary parts is needed to generate any $T$-odd structure. The most straightforward option is to go beyond the tree-level gluon correlator by including the interference with the one-gluon exchange process (in eikonal approximation). The latter represents the first-order approximation of the gauge link operator. The main consequence of this strategy is that the calculated $T$-odd TMDs is gauge-link sensitive, and thus process dependent. The two linearity functions corresponding to the $f$-type gauge link are obtained by suitably projecting the transverse part of the corresponding gluon correlator, and they are equal up to a minus sign

$$
h_{1}^{g[+,+]}\left(x, \boldsymbol{p}_{T}^{2}\right) \equiv-h_{1}^{g[-,-]}\left(x, \boldsymbol{p}_{T}^{2}\right) .
$$

In our preliminary analysis we use a simplified expression of the nucleon-gluon-spectator vertex, where the $g_{2}$ form factor in Eq. (1) is set to zero. For the sake of consistency, we fitted again model parameters to NNPDF results by using the simplified formula for the vertex. Fig. 1 shows the $\boldsymbol{p}_{T}^{2}$-shape of the $[+,+]$ linearity function at $x=10^{-1}$ and at $x=10^{-3}$, and at the initial scale $Q_{0}=1.64 \mathrm{GeV}$. The absolute value of our density is clearly a non-Gaussian function of $\boldsymbol{p}_{T}^{2}$, with a large flattening tail at large $\boldsymbol{p}_{T}^{2}$-values and a small nonzero value when $\boldsymbol{p}_{T}^{2}$ goes to zero. We remark that this behavior could radically change when the full-vertex calculation will become available. 


\section{Closing statements}

We have extended our spectator-model framework by including a preliminary version of the $f$-type gluon linearity TMD function. Once the calculation of all the $T$-odd gluon TMDs will be completed, we will include standard TMD evolution and perform phenomenological analyses on the relevant spin asymmetries that can be studied at new-generation colliding facilities, as the Electron-Ion Collider (EIC) [43], the High-Luminosity Large Hadron Collider (HL-LHC) [44], NICA-SPD [45], and the Forward Physics Facility (FPF) [46].

\section{References}

[1] P. J. Mulders J. Rodrigues, Phys. Rev. D63, 094021 (2001), hep-ph/0009343.

[2] S. Meissner, A. Metz, K. Goeke, Phys. Rev. D76, 034002 (2007), hep-ph/0703176.

[3] C. Lorce' B. Pasquini, JHEP 09, 138 (2013), 1307 . 4497.

[4] D. Boer, S. Cotogno, T. van Daal, P. J. Mulders, A. Signori, Y.-J. Zhou, JHEP 10, 013 (2016), 1607.01654.

[5] Z. Lu B.-Q. Ma, Phys. Rev. D94, 094022 (2016), 1611.00125.

[6] J.-P. Lansberg, C. Pisano, F. Scarpa, M. Schlegel, Phys. Lett. B 784, 217 (2018), [Erratum: Phys.Lett.B 791, 420-421 (2019)], 1710.01684.

[7] D. Gutierrez-Reyes, S. Leal-Gomez, I. Scimemi, A. Vladimirov, JHEP 11, 121 (2019), 1907. 03780.

[8] F. Scarpa, D. Boer, M. G. Echevarria, J.-P. Lansberg, C. Pisano, M. Schlegel, Eur. Phys. J. C 80, 87 (2020), 1909. 05769.

[9] C. Adolph et al. (COMPASS), Phys. Lett. B 772, 854 (2017), 1701.02453.

[10] U. D’Alesio, F. Murgia, C. Pisano, P. Taels, Phys. Rev. D96, 036011 (2017), 1705.04169.

[11] U. D’Alesio, C. Flore, F. Murgia, C. Pisano, P. Taels, Phys. Rev. D 99, 036013 (2019), 1811.02970.

[12] U. D’Alesio, F. Murgia, C. Pisano, P. Taels, Phys. Rev. D100, 094016 (2019), 1908.00446.

[13] S. J. Brodsky, D. S. Hwang, I. Schmidt, Phys. Lett. B530, 99 (2002), hep-ph/0201296.

[14] J. C. Collins, Phys. Lett. B 536, 43 (2002), hep-ph/0204004.

[15] X.-d. Ji F. Yuan, Phys. Lett. B 543, 66 (2002), hep-ph/0206057.

[16] C. Bomhof, P. Mulders, F. Pijlman, Eur. Phys. J. C 47, 147 (2006), hep-ph/0601171.

[17] T. C. Rogers, Phys. Rev. D 88, 014002 (2013), 1304.4251.

[18] A. D. Bolognino, F. G. Celiberto, D. Yu. Ivanov, A. Papa, Eur. Phys. J. C78, 1023 (2018), 1808.02395. 
[19] A. D. Bolognino, F. G. Celiberto, D. Yu. Ivanov, A. Papa, Frascati Phys. Ser. 67, 76 (2018), 1808.02958.

[20] A. D. Bolognino, F. G. Celiberto, D. Yu. Ivanov, A. Papa, Acta Phys. Polon. Supp. 12, 891 (2019), 1902.04520.

[21] A. D. Bolognino, A. Szczurek, W. Schaefer, Phys. Rev. D 101, 054041 (2020), 1912.06507.

[22] F. G. Celiberto, Nuovo Cim. C42, 220 (2019), 1912 . 11313.

[23] F. G. Celiberto, D. Gordo Gómez, A. Sabio Vera, Phys. Lett. B786, 201 (2018), 1808 . 09511.

[24] F. G. Celiberto, Eur. Phys. J. C 81, 691 (2021), 2008. 07378.

[25] A. D. Bolognino, F. G. Celiberto, M. Fucilla, D. Yu. Ivanov, A. Papa, Eur. Phys. J. C 79, 939 (2019), 1909.03068.

[26] A. D. Bolognino, F. G. Celiberto, D. Yu. Ivanov, A. Papa, W. Schäfer, A. Szczurek, Eur. Phys. J. C 81, 846 (2021), 2107.13415.

[27] F. G. Celiberto, D. Yu. Ivanov, M. M. A. Mohammed, A. Papa, Eur. Phys. J. C 81, 293 (2021), 2008.00501.

[28] F. G. Celiberto, D. Yu. Ivanov, A. Papa, Phys. Rev. D 102, 094019 (2020), 2008 . 10513.

[29] A. D. Bolognino, F. G. Celiberto, M. Fucilla, D. Yu. Ivanov, A. Papa, Phys. Rev. D 103, 094004 (2021), 2103.07396.

[30] F. G. Celiberto, M. Fucilla, D. Yu. Ivanov, A. Papa, Eur. Phys. J. C 81, 780 (2021), 2105. 06432.

[31] F. G. Celiberto, M. Fucilla, D. Yu. Ivanov, M. M. A. Mohammed, A. Papa, Phys. Rev. D 104, 114007 (2021), 2109. 11875.

[32] F. G. Celiberto M. Fucilla (2022), 2202 . 12227.

[33] M. Nefedov, Phys. Rev. D 104, 054039 (2021), 2105 . 13915.

[34] M. Hentschinski, Phys. Rev. D 104, 054014 (2021), 2107.06203.

[35] A. Bacchetta, F. Conti, M. Radici, Phys. Rev. D78, 074010 (2008), 0807.0323.

[36] A. Bacchetta, M. Radici, F. Conti, M. Guagnelli, Eur. Phys. J. A45, 373 (2010), 1003.1328.

[37] A. Bacchetta, F. G. Celiberto, M. Radici, P. Taels, Eur. Phys. J. C 80, 733 (2020), 2005 . 02288.

[38] A. Bacchetta, F. G. Celiberto, M. Radici, P. Taels, in DIS 2021 (2021), 2107.13446.

[39] F. G. Celiberto, Nuovo Cim. C44, 36 (2021), 2101.04630.

[40] A. Bacchetta, F. G. Celiberto, M. Radici (2021), 2111.01686.

[41] R. D. Ball, V. Bertone, M. Bonvini, S. Marzani, J. Rojo, L. Rottoli, Eur. Phys. J. C78, 321 (2018), 1710.05935.

[42] E. R. Nocera, R. D. Ball, S. Forte, G. Ridolfi, J. Rojo (NNPDF), Nucl. Phys. B887, 276 (2014), 1406.5539.

[43] R. Abdul Khalek et al. (2021), 2103.05419.

[44] E. Chapon et al., Prog. Part. Nucl. Phys. 122, 103906 (2022), 2012 . 14161.

[45] A. Arbuzov et al., Prog. Part. Nucl. Phys. 119, 103858 (2021), 2011.15005.

[46] L. A. Anchordoqui et al. (2021), 2109. 10905. 